\title{
Alpha-synuclein is involved in manganese-induced spatial memory and synaptic plasticity impairments via TrkB/Akt/Fyn-mediated phosphorylation of NMDA receptors
}

\author{
Zhuo Ma ${ }^{1}$, Kuan Liu', Xin-Ru Li', Can Wang ${ }^{1}$, Chang Liu', Dong-Ying Yan', Yu Deng ${ }^{1}$, Wei Liu ${ }^{1}$ and Bin Xu (1)
}

\begin{abstract}
Manganese $(\mathrm{Mn})$ overexposure produces long-term cognitive deficits and reduces brain-derived neurotrophic factor (BDNF) in the hippocampus. However, it remains elusive whether Mn-dependent enhanced alpha-synuclein (a-Syn) expression, suggesting a multifaceted mode of neuronal toxicities, accounts for interference with BDNF/TrkB signaling. In this study, we used C57BL/6J WT and a-Syn knockout (KO) mice to establish a model of manganism and found that Mn-induced impairments in spatial memory and synaptic plasticity were related to the a-Syn protein. In addition, consistent with the long-term potentiation (LTP) impairments that were observed, a-Syn KO relieved Mn-induced degradation of PSD95, phosphorylated CaMKIla, and downregulated SynGAP protein levels. We transfected HT22 cells with lentivirus (LV)-a-Syn shRNA, followed by BDNF and Mn stimulation. In vitro experiments indicated that a-Syn selectively interacted with TrkB receptors and inhibited BDNF/TrkB signaling, leading to phosphorylation and downregulation of GluN2B. The binding of a-Syn to TrkB and Fyn-mediated phosphorylation of GluN2B were negatively regulated by BDNF. Together, these findings indicate that Mn-dependent enhanced a-Syn expression contributes to further exacerbate BDNF protein-level reduction and to inhibit TrkB/Akt/Fyn signaling, thereby disturbing Fyn-mediated phosphorylation of the NMDA receptor GluN2B subunit at tyrosine. In KO a-Syn mice treated with Mn, spatial memory and LTP impairments were less pronounced than in WT mice. However, the same robust neuronal death was observed as a result of Mn-induced neurotoxicity.
\end{abstract}

\section{Introduction}

Manganese $(\mathrm{Mn})$ is an essential nutrient because it is required for cellular function in many metabolic pathways. However, the central nervous system (CNS) is highly vulnerable to Mn toxicity, which leads to many sensory disturbances and neurobehavioral deficiency. Mn toxicity can also manifest as a disease called manganism, which shares symptoms that are similar to those of Parkinson's disease $(\mathrm{PD})^{1}$. Manganism is characterized by

\footnotetext{
Correspondence: Bin Xu (bxu10@cmu.edu.cn)

'Department of Environmental Health, School of Public Health, China Medical University, No. 77 Puhe Road, Shenyang North New Area, 110122 Shenyang, Liaoning Province, People's Republic of China

Edited by A. Verkhratsky
}

rigidity, bradykinesia, emotional instability, and intellectual and memory impairment at an early stage. Environmental and occupational Mn exposure occurs mainly by welding, mining, smelters, lacquers, dry cell batteries, and Mn-rich agrochemicals ${ }^{2}$. Bailey ${ }^{3}$ reported that Mn overexposure during development produces long-term cognitive deficits and reduces brain-derived neurotrophic factor (BDNF) in the hippocampus. Although the neurotoxic mechanisms of Mn such as oxidative stress, energy failure, and the disturbance of neurotransmitter metabolism are well-elucidated ${ }^{4}$, the mechanism underlying these spatial learning and memory deficits is unclear.

BDNF, acting through the receptor tropomyosin kinase receptor $\mathrm{B}(\operatorname{Trk} \mathrm{B})$, is considered a key molecule in the 
neurobiological mechanisms involved in learning and memory ${ }^{5}$. BDNF binding to TrkB triggers its dimerization through conformational changes and autophosphorylation of tyrosine residues in its intracellular domain (ICD), activating mitogen-activated protein kinase (MAPK), phosphatidylinositol 3-kinase (PI3K), and phospholipase C-g1 (PLC- $\gamma 1$ ) signaling pathways, and mediating neural differentiation, survival, and neurogenesis $^{6}$. However, Kang ${ }^{7}$ found that $\alpha$-synuclein ( $\alpha$-Syn), when overexpressed, selectively interacts with $\operatorname{Trk} B$ receptors and inhibits $\mathrm{BDNF} / \mathrm{TrkB}$ signaling, leading to dopaminergic neuronal death. $\alpha$-Syn localizes to presynaptic terminals nearby synaptic vesicles and is predominantly expressed in the neurons of the CNS. Our previous research found that $\mathrm{Mn}$-dependent enhanced $\alpha$ Syn expression leads to neuronal injury ${ }^{8}$. In addition, $\mathrm{Mn}$ exposure promotes $\alpha$-Syn secretion in exosomal vesicles. The overexpression of $\alpha$-Syn can disrupt synaptic function, resulting in cognitive disturbance ${ }^{9}$. Therefore, we speculate that the overexpression of $\alpha$-Syn and its subsequent interference with BDNF/TrkB signaling may play an important role in linking the complicated neurobehavioral deficiency observed following $\mathrm{Mn}$ exposure, although the molecular mechanism of this process remains elusive.

In this study, we provided evidence that Mn-induced spatial learning and memory deficits are related to $\alpha$-Syn disturbing the BDNF/TrkB neurotrophic signaling pathways in vivo and in vitro. Additionally, we used shRNA to knockdown $\alpha$-Syn and found that BDNF/TrkB signaling pathways exhibited a certain recovery. Hence, this innovative finding provides insight into a pathological role(s) of $\alpha$-Syn in mediating BDNF/TrkB signal transduction and may represent a relatively unknown mechanism by which $\mathrm{Mn}$-dependent enhanced $\alpha$-Syn expression is responsible for learning and memory impairments in mice.

\section{Materials and methods Chemicals}

Manganese (II) chloride tetrahydrate, BDNF, and DuoLink proximity ligation assay (PLA) reagent were purchased from Sigma Chemical Co. (St. Louis, MO, USA). The pGCSIL-GFP- $\alpha$-Syn small hairpin RNA (shRNA) ${ }^{10}$ was purchased from Shanghai GeneChem Co. Ltd. (Shanghai, China). A Cell Counting Kit-8 (CCK-8) assay kit and lactate dehydrogenase (LDH) kit were purchased from Beyotime Biotech Co. Ltd. (China). $\alpha$-Syn primary antibody was obtained from Thermo Fisher Scientific. PSD95, SynGAP, $\beta$-actin, phospho-CaMKII (Thr286), CaMKII- $\alpha$, NMDA receptor 2A (GluN2A), NMDA receptor 2B (GluN2B), Fyn, phospho-NMDA receptor 2A (GluN2A) (Tyr1246), and phospho-NMDA receptor 2B (GluN2B) (Tyr1472) primary antibodies were purchased from Cell Signaling Technology. TrkB, AKT, and AKTphospho-S473 primary antibodies were purchased from Proteintech Biotech Co. Ltd. (China). BDNF and phospho-Fyn (Y530) were purchased from Abcam Ltd. (Hong Kong). Secondary antibodies including horseradish peroxidase (HRP)-conjugated anti-rabbit and HRPconjugated anti-mouse were purchased from Abcam Ltd. (Hong Kong).

\section{Animals and treatments}

Homozygous $\alpha$-Syn gene knockout (KO) male mice were crossed with wild-type (WT) female mice (C57BL/ 6J) (Liaoning Changsheng Biotechnology Co., Ltd. SCXK 2015-0003; Benxi City, China) maintained in a stable breeding colony ${ }^{8}$.

Ten-week-old homozygous WT and $\alpha$-Syn KO mice $(25 \pm 5 \mathrm{~g})$ from an identical offspring generation of F7 heterozygotes were used in the in vivo experiments. The WT and $\alpha$-Syn KO mice were consolidated and then randomly divided into four groups $(n=10$ per group) (female: male $=1: 1$ ): WT control, $\mathrm{KO}$ control, WT Mn treatment $\left(100 \mu \mathrm{mol} / \mathrm{kg} \mathrm{MnCl}_{2}\right)$, and $\mathrm{KO} \mathrm{Mn}$ treatment $\left(100 \mu \mathrm{mol} / \mathrm{kg} \mathrm{MnCl}_{2}\right)$. The control group mice were intraperitoneally (i.p.) injected with normal saline. $\mathrm{MnCl}_{2}$ treated mice were i.p. injected with $100 \mu \mathrm{mol} / \mathrm{kg}$ $\mathrm{MnCl}_{2} \cdot 4 \mathrm{H}_{2} \mathrm{O}$ in normal saline five times per week consecutively for 6 weeks. Animals were housed in conventional enclosures at $24 \pm 1{ }^{\circ} \mathrm{C}$ under a $12 \mathrm{~h}$ light/dark cycle. Six mice per group were chosen as the minimal number of animals needed for carrying out a comparative study. Blinding was performed in the experimental procedures and the data analysis.

\section{Morris water maze (MWM) test}

The MWM test was performed to estimate the capacity of spatial learning and memory, as previously described ${ }^{11}$. The test was composed of a circular tank $(120 \mathrm{~cm}$ in diameter, $50 \mathrm{~cm}$ in height) filled with $24{ }^{\circ} \mathrm{C}$ water colored with white powder, and an escape platform placed $1.5 \mathrm{~cm}$ below the water surface. Some geometric images were fixed around the tank to assist the swimming mice with their orientation, and a video camera monitored the trajectory and transmitted the data to a computer. The mice were placed into the pool from any quadrant, and they were able to swim freely until they reached the platform. This was repeated four times per day, and after 6 days of training, latency, mean speed, and mean distance were analyzed in a probe test phase, and speed, crossing times, and time in the target quadrant were acquired after $48 \mathrm{~h}$.

\section{Electrophysiology}

The mouse brains were carefully placed in the chilled artificial cerebral spinal fluid (aCSF) buffer, including $124 \mathrm{mM} \mathrm{NaCl}, 3 \mathrm{mM} \mathrm{KCl}, 26 \mathrm{mM} \mathrm{NaHCO}_{3}, 2 \mathrm{mM} \mathrm{CaCl}_{2}$, 
$1 \mathrm{mM} \mathrm{MgSO} 4,1.25 \mathrm{mM} \mathrm{KH}_{2} \mathrm{PO}_{4}$, and $10 \mathrm{mM}$ D-glucose with $95 \% \mathrm{O}_{2}$ and $5 \% \mathrm{CO}_{2}$ gas. Then, the hippocampus was cut into 300-nm thick slices and incubated in aCSF buffer for $2 \mathrm{~h}$. These brain slices were secured with a metal probe in an $8 \times 8$ array microelectrode chamber, and a MED64 Mobius (Alpha MED Scientific, Japan) microelectrode array system (MEA) was used to record the long-term potentiation (LTP). The test-stimulation intensity was placed in the CA1-CA3 region and set to elicit $30 \%$ (or $50 \%$ ) of the maximum field excitatory postsynaptic potential (fEPSP) response according to the I/O (input/output) curve. After $20 \mathrm{~min}$, the baseline was recorded, and LTP was induced by a $100 \mathrm{~Hz} / \mathrm{s}$ highfrequency stimulus that was repeated three times, and the fEPSP slope change was recorded for another $60 \mathrm{~min}^{12}$.

\section{Nissl staining}

After internal fixation with 4\% formaldehyde overnight, the mouse brains were quickly removed and fixed with paraformaldehyde for 3 days. Then, the samples were transferred into $70 \%, 80 \%, 90 \%, 95 \%$, and $100 \%$ ethyl alcohol (12 h for each) and dimethylbenzene for $5 \mathrm{~min}$ for dehydration. The treated brains were embedded into the paraffin and sliced into 8- $\mu \mathrm{m}$-thick hippocampus sections. After dewaxing and hydration, these slices were stained with toluidine blue stain (DK0023, Beijing Leagene Biotechnology Co., Ltd.) for $30 \mathrm{~min}$ in a dark and humid box at $60^{\circ} \mathrm{C}$. Finally, slices were immersed in $95 \%$ ethanol for $1 \mathrm{~min}$ and dimethylbenzene for $15 \mathrm{~min}$ and sealed with neutral balsam. The slices were observed with a ScanScope scanner system (view magnification: $\times 400$; Leica, USA) and analyzed by ImageScope $12.0^{13}$.

\section{Transmission electron microscopy}

The neural synapse ultrastructure was observed by transmission electron microscopy according to a protocol from a previous study ${ }^{14}$. Briefly, the brain was fixed in $2.5 \%$ glutaraldehyde for $2 \mathrm{~h}$ and then postfixed in $1 \%$ osmium tetroxide for another $2 \mathrm{~h}$. After dehydration in ethyl alcohol gradients, the sample was embedded, sectioned, and stained with uranyl acetate and lead citrate. Finally, a transmission electron microscope (H-7650, Hitachi) was used for observation at $\times 40,000$ magnification. The thickness of postsynaptic density was analyzed using image analysis software (Image 1.42q, USA).

\section{Measurement of $\mathrm{Mn}$ concentration}

The hippocampus was dissected from the mouse brain, weighed, and digested in $500 \mu \mathrm{l} 70 \% \mathrm{HNO}_{3}$ for the metaltrack assay. After partial evaporation, the samples were cooled to room temperature, $500 \mu \mathrm{l}$ of $\mathrm{H}_{2} \mathrm{O}_{2}$ (36.5-38.0\% for trace metal analysis) was added, and the solution was completely evaporated. Then, the precipitate was dissolved in $5 \mathrm{~mL}$ of deionized water and analyzed on a
Hitachi $180-80$ atomic absorption spectrophotometer (Hitachi Ltd., Tokyo, Japan) $^{14}$.

\section{Cell culture and treatments}

Mouse hippocampal neuronal cell line HT22 was purchased from the Shanghai iCell Biotechnology Company. HT22 cells were cultured in Dulbecco's modified Eagle's medium (DMEM, Gibco, Cat. no. 10566016) with 10\% fetal bovine serum maintained in a humidified atmosphere with $95 \%$ air and $5 \% \mathrm{CO}_{2}$ at $37^{\circ} \mathrm{C}$. Then, cells were differentiated in DMEM medium containing $1 \times$ $\mathrm{N} 2$ supplement, $50 \mathrm{ng} / \mathrm{ml}$ nerve growth factor- $\beta$ (NGF- $\beta$, Gibco, Cat. no. 17502001), $100 \mu \mathrm{M}$ phorbol 12,13-dibutyrate (Sigma-Aldrich, Cat. no. P1269), and $100 \mu \mathrm{M}$ dibutyryl cAMP (Millipore, Cat. no. 28745) for 24 h before use $^{15}$. For knockdown of $\alpha$-Syn, shRNA was designed (Gene Bank Accession No: AF179273) ${ }^{16}$, and lentiviral vector construction and transfection were performed as previously described ${ }^{17}$. The in vitro experiment was divided into three parts, of which the first part was set up with six groups as follows: three control groups (DMEM control, lentivirus (LV)-Syn shRNA group, and LVscrambled shRNA group) and three $100 \mu \mathrm{M}$ Mn-treated groups (Mn group, LV-Syn shRNA + Mn group, and LVscrambled shRNA + Mn group). For the second part, pretreatment with BDNF was performed. Five groups were divided as follows: control group; Mn $(100 \mu \mathrm{M})$ group; BDNF ( $80 \mathrm{ng} / \mathrm{ml})$ control group; BDNF $(40 \mathrm{ng} / \mathrm{ml})$ $+\mathrm{Mn}$ group; and BDNF $(80 \mathrm{ng} / \mathrm{ml})+\mathrm{Mn}$ group. For the last part, we combined the first part with the second part. HT22 cells and HT22 cells transfected with LV-Syn shRNA were divided into the control group, $\mathrm{Mn}(100 \mu \mathrm{M})$ group, BDNF $(80 \mathrm{ng} / \mathrm{ml})$ group, and BDNF $(80 \mathrm{ng} / \mathrm{ml})$ + Mn group.

\section{Cell viability and LDH assay}

The cellular viability was evaluated by the CCK- 8 assay (CCK-8 Kit, Beyotime, No: C0037, China), and the level of LDH release induced by $\mathrm{MnCl}_{2}$ was evaluated by assay with an LDH Kit (Beyotime, No: C0016, China). HT22 cells were seeded onto 96-well plates and treated with $100 \mu \mathrm{M}$ Mn or pretreated with LV-Syn shRNA, LVscrambled shRNA, or BDNF. After $24 \mathrm{~h}$ of culture, the cells were assayed with the CCK- 8 and LDH kits according to the manufacturer's protocol using a microplate reader (Biotech Synergy H1, USA).

\section{Western blot analysis}

The expression of the protein was evaluated by immunoblotting according to a previously published method ${ }^{18}$. Briefly, total proteins were extracted from the hippocampus and HT22 cells according to standard procedures. Quantified protein $(20 \mu \mathrm{g})$ was separated via $8 \%, 10 \%$, or $15 \%$ sodium dodecyl sulfate-polyacrylamide gel 
electrophoresis (SDS-PAGE). Then, the samples were transferred to PVDF membranes, blocked in 5\% bovine serum albumin (BSA) for $2 \mathrm{~h}$, and incubated with the appropriate primary antibodies overnight at $4{ }^{\circ} \mathrm{C}$. These important primary antibodies are listed as follows: PSD95 (1:1000, Cell Signaling Technology, Cat. no. \#2507), SynGAP (1:1000, Cell Signaling Technology, Cat. no. \#3200), TrkB (1:500, Proteintech, Cat. no. 13129), alphaSynuclein (1:500, Invitrogen, Cat. no. \#MA5-12272), $\beta$-actin (1:1000, Cell Signaling Technology, Cat. no. \#8457), BDNF (1:1000, Abcam, ab182199), CaMKII- $\alpha$ (1:1000, Cell Signaling Technology, Cat. no. \#3357), Phospho-CaMKII (Thr286) (1:1000, Cell Signaling Technology, Cat. no. \#12716), NMDA Receptor 2A (GluN2A) (1:1000, Cell Signaling Technology, Cat. no. \#4205), NMDA Receptor 2B (GluN2B) (1:1000, Cell Signaling Technology, Cat. no. \#4207), AKT (1:500, Proteintech, Cat. no. 10176), AKT-phospho-S473 (1:3000, Proteintech, Cat. no. 66444), Fyn (1:1000, Cell Signaling Technology, Cat. no. \#4023), Anti-Fyn (phospho Y530) (1:500, Abcam, ab182661), Phospho-NMDA Receptor 2A (GluN2A) (Tyr1246) (1:1000, Cell Signaling Technology, Cat. no. \#4206), and Phospho-NMDA Receptor 2B (GluN2B) (Tyr1472) (1:1000, Cell Signaling Technology, Cat. no. \#4208). Subsequently, the membranes were incubated with matched secondary antibodies for $2 \mathrm{~h}$. The results were detected using enhanced chemiluminescence (ECL) and analyzed using image analysis software (Image) 1.42q, USA).

\section{Alpha-synuclein western blots}

Briefly, after extracted and normalized the total protein from the hippocampus, the samples without boiling were separated by $4-20 \%$ non-denaturing polyacrylamide-gradient gel electrophoresis (Thermo Fisher Scientific, USA). Then, the samples were transferred to PVDF membranes and incubated with the alpha-Synuclein antibody (1:500, Invitrogen, Cat. no. \#MA5-12272). After 24 h, the membranes were incubated with a matched secondary antibody for $2 \mathrm{~h}$. The results were detected using ECL and analyzed using image analysis software (Image) 1.42q, USA).

\section{Co-immunoprecipitation (Co-IP) of alpha-synuclein with TrkB}

HT22 cells were harvested in RIPA buffer with $1 \mathrm{mM}$ phenylmethylsulfonyl fluoride (PMSF) and centrifuged at $12,000 \times g$ for $15 \mathrm{~min}$ at $4{ }^{\circ} \mathrm{C}$. Then, the protein was normalized and mixed with $4 \mu \mathrm{l}$ of anti-alpha-synuclein antibody (Invitrogen, Cat. no. MA5-12272) or rabbit IgG antibody at $4{ }^{\circ} \mathrm{C}$. The following day, $25 \mu$ Protein A/G magnetic beads were reacted with the sample/antibody complex for $2 \mathrm{~h}$ at $4{ }^{\circ} \mathrm{C}$. The supernatant was separated by $8 \%$ or $12 \%$ SDS-PAGE, and then analyzed by western blot assay ${ }^{18}$. The primary antibody TrkB was purchased from Proteintech Biotech Co. Ltd (China, Cat. no. 13129).

\section{Proximity ligation assay}

Pretreated HT22 cells were assayed using DuoLink PLA according to the manufacturer's protocol ${ }^{19}$. Negative control sections were prepared using mouse $\operatorname{IgG}$ for $\alpha$ Syn and rabbit immunoglobulin fraction for TrkB. Then, the signal strength of the red points in each cell was calculated and analyzed using a confocal microscope (excitation: $594 \mathrm{~nm}$, emission: $624 \mathrm{~nm}$, magnification: $\times 60$ ). NIS-Elements Viewer 4.50 software (Nikon, Japan) was used to measure the signaling level.

\section{Statistical analysis}

Statistical analyses were performed with GraphPad Prism 8.0.1 (GraphPad Software, CA, USA). Two-way ANOVA and the SNK-q were used to assess the effect of the $\mathrm{Mn}$ factor and genotype factor in vivo. For the in vitro experiments, one-way ANOVA and the SNK-q test were used to analyze the significant variances: $P$ values of less than 0.01 or 0.05 were set as statistically significant. All experiments were repeated at least three replicated times. " $\mathrm{n}$ " refers to samples obtained from different mice in vivo, and parallel samples in vitro. Bars represent mean \pm SD.

\section{Results}

Mn impairs spatial memory and synaptic plasticity in WT and a-Syn KO mice

To investigate the effect of $\mathrm{Mn}$ on spatial learning and memory in the presence of $\alpha$-Syn protein, we tested the reference memory of mice in the MWM (Fig. 1a). In the acquisition training phase, between the WT and $\alpha$-Syn KO control groups, the escape latencies, mean speed, and mean distance exhibited no significant differences (Fig. 1b-e). However, Mn-treated mice showed significantly increased times for escape latency and mean distance compared with control groups, which indicated learning and memory impairments. For the two Mn-treated groups, the data analysis showed that $\alpha$-Syn KO mice performed better than WT mice with Mn treatment, as revealed by a significant decrease in escape latency and mean distance compared with Mn-treated WT mice on the 6th day (escape latency, $F$ (Mn factor) $=153.725, P=0.00, F$ (genotype) $=1.995, P>0.05$, $F_{\text {(genotype vs. Mn interaction) }}=25.772, P=0.00$; mean distance, $F_{(\text {Mn factor })}=67.656, P=0.00, F_{\text {(genotype) }}=2.332, P>0.05$, $F$ (genotype vs. Mn interaction) $=6.491, P=0.019$ ).

Then, $48 \mathrm{~h}$ after the acquisition sessions, the platform was removed. In the probe test, similar crossing times in the platform (target) quadrant were measured for the two control groups, and they spent similar amounts of time in the target quadrant. However, Mn-treated mice showed significantly decreased crossing times and time in the target quadrant during the removal testing session, 
a Morris water maze(MWM)

Electrophysiology

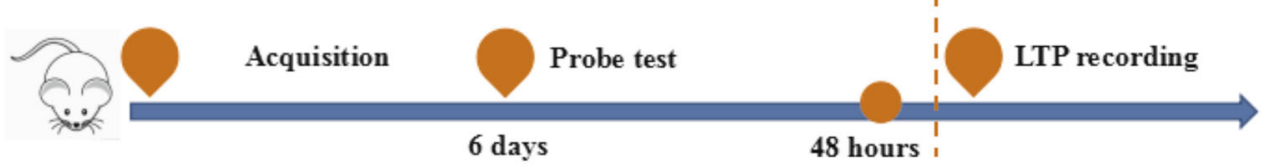

b

WT control

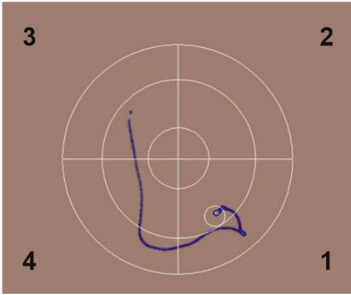

KO control

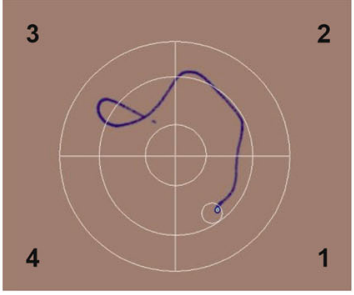

WT Mn

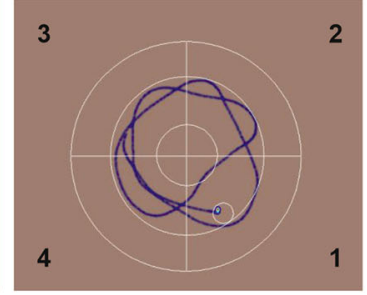

KO Mn

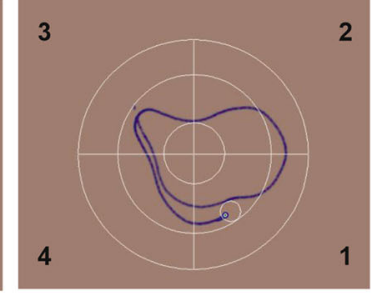

- WT control

- KO control

A. WT Mn

- $-\mathrm{KO} \mathrm{Mn}$

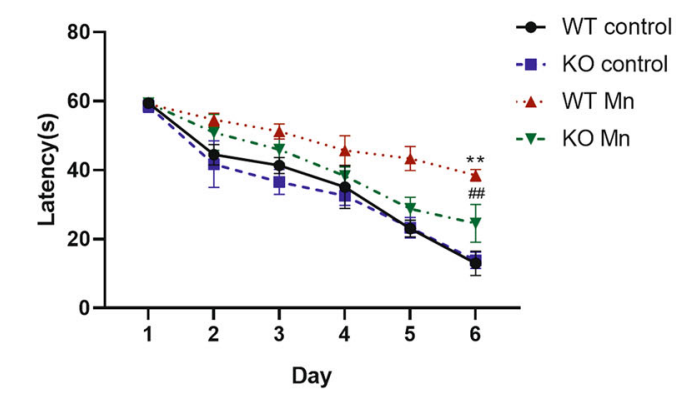

e

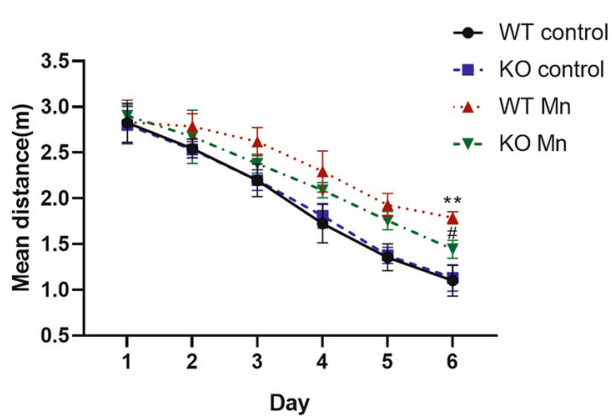

g

- WT control

- KO control

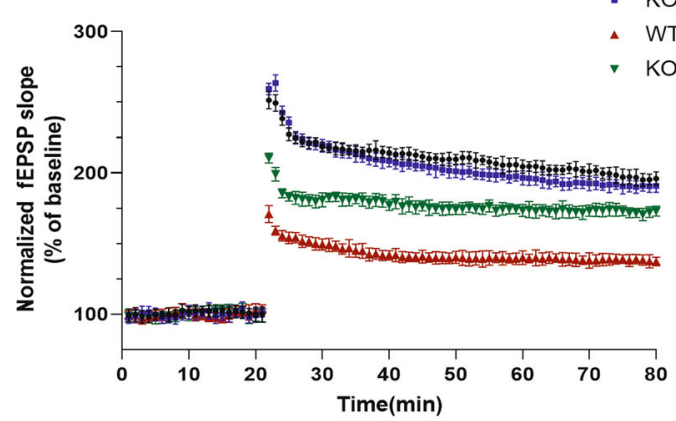

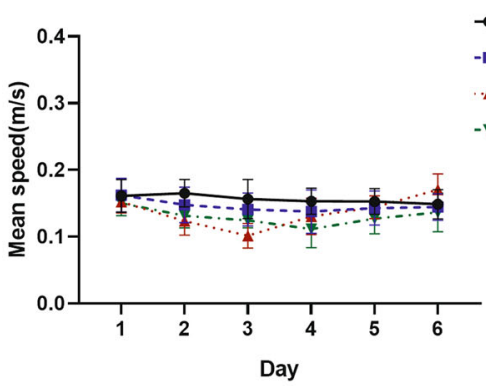

$48 \mathrm{~h}$

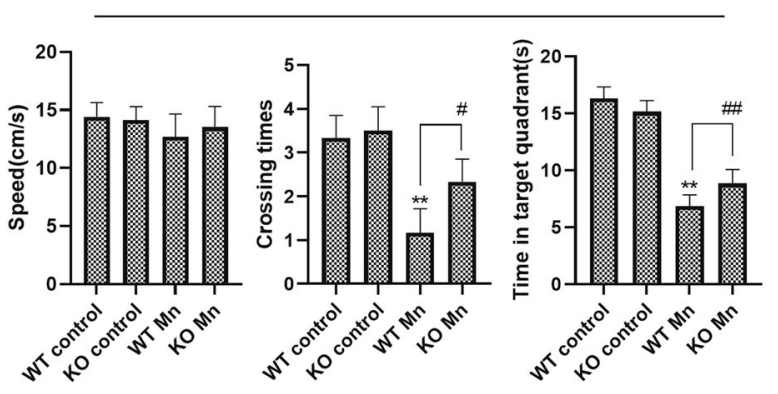

Fig. $1 \mathrm{Mn}$ impairs the spatial memory in WT and a-Syn KO mice. a The experimental procedure for Morris water maze (MWM) and electrophysiology tests. b A representative searching trace on the 6th day of the training phase. c-e The escape latency, mean speed, and mean distance to reach the hidden platform. $\mathbf{f}$ After $48 \mathrm{~h}$, the mice were tested again with the platform removed, and the speed (left), crossing times (middle), and time in the target quadrant (right) were recorded. $\mathbf{g}, \mathbf{h}$ The normalized fEPSP slope (\% of baseline) recorded from the CA1-CA3 region in hippocampal slices. $n=6$. ${ }^{*} P<0.01$ compared to controls; ${ }^{\#} P<0.01$, and ${ }^{\#} P<0.05$ for comparison between the Mn-treated WT mice. 
compared with control groups (Fig. 1f). In addition, Mntreated $\alpha$-Syn KO mice were relieved of the effect of Mn. (crossing times, $F$ (Mn factor) $=47.619, P=0.00$, $F$ (genotype) $=3.619, P>0.05, F$ (genotype vs. Mn interaction) $=$ 4.286, $P=0.05$; time in the target quadrant, $F_{(\mathrm{Mn} \text { factor })}=$ $333.871, P=0.00, F$ (genotype) $=0.915, P>0.05, F$ (genotype vs. Mn interaction) $=13.265, P=0.002$ ). Our data suggested that $\alpha$-Syn $\mathrm{KO}$ ameliorated the learning and memory impairments induced by Mn.

Deficits in LTP involved in learning and memory may contribute to a number of neurological diseases. In the hippocampus CA3-CA1 region, our results showed that LTP was weakened in Mn-treated mice compared with the control mice, with the maximum inhibition appearing in Mn-treated WT mice. The fEPSP slope change was used to reflect the level of LTP formation. Figure $1 \mathrm{~g}$, $\mathrm{h}$ indicates that there were no significant differences in the increasing rate of fEPSP slopes between control WT and $\alpha$-Syn KO mice, as indicated by the changes in the postsynaptic potential slopes. Mn-treated WT mice showed a decreased fEPSP slope growth rate compared with control mice. In addition, $\alpha$-Syn knockout weakened the effect of Mn (fEPSP slope at $80 \mathrm{~min}, F$ (Mn factor) $=121.913, P=$ $0.00, F$ (genotype) $^{2.811,} P>0.05, \quad F$ (genotype vs. Mn interaction) $=209.082, P=0.00$ ). These data reinforced the idea that Mn-induced impairments in spatial memory and synaptic plasticity were related to LTP.

\section{Mn induces synaptic degeneration in WT and a-Syn KO mice}

Nissl staining showed that the number of surviving neurons was decreased in the CA1 region in Mn-treated WT and $\alpha$-Syn KO mice compared with their control mice (WT control $150.1 \pm 10.401$ vs. WT Mn $94.66 \pm$ 12.662; $\mathrm{KO}$ control $154.0 \pm 9.585$ vs. $\mathrm{KO} \mathrm{Mn} 99.34 \pm$ $11.500, F_{\text {(Mn factor })}=70.668, P=0.00, F_{\text {(genotype) }}=0.439$, $P>0.05, F$ (genotype vs. Mn interaction) $=0.003, P>0.05$, Fig. 2a, b). We used transmission electron microscopy (TEM) to observe the pathological features of synaptic ultrastructure. In WT and $\alpha$-Syn KO mice, normal synaptic ultrastructure was observed in the hippocampus, consisting of a clear synaptic cleft and postsynaptic density (PSD). Electron microscopy data were quantitated and showed that the PSD thickness in the synapses decreased, and the synaptic cleft became blurred in the Mn-treated WT and $\alpha$-Syn KO mice (WT control $100 \% \pm 0.061$ vs. WT Mn $51.6 \% \pm 0.080$; KO control $100 \% \pm 0.065$ vs. KO Mn $\quad 80.8 \% \pm 0.056)$. However, $\alpha$-Syn knockout relieved Mn-induced impairment of PSD thickness $\left(F_{(\mathrm{Mn}}\right.$ factor) $=1509.49, P=0.00, F$ (genotype) $_{\text {(n) }}=1.068, P>0.05$, $F$ (genotype vs. Mn interaction) $=26.833, P=0.00$, Fig. 2 c, d).

We measured Mn concentration in the hippocampus to determine how much was absorbed in Mn-treated mice. Statistical comparisons revealed a significant increase in
Mn-treated WT and $\alpha$-Syn KO mice compared with their control counterparts (5.25- and 4.55-fold, respectively, $F$ $($ Mn factor $)=234.795, P=0.00, F$ (genotype) $=0.081, P>0.05$, $F$ (genotype vs. Mn interaction) $=0.257, P>0.05$ ). In addition, between Mn-treated WT and $\alpha$-Syn KO mice, statistical analysis showed no difference in $\mathrm{Mn}$ concentration (Fig. 2e).

To further investigate synaptic degeneration, we examined the levels of postsynaptic proteins (PSD95, CaMKII $\alpha$, and SynGAP) in the hippocampus of WT and $\alpha$-Syn $\mathrm{KO}$ mice. After treatment with $\mathrm{Mn}$, the significant decrease in the level of PSD95 associated with increases in the levels of phosphorylated CaMKII $\alpha$ and SynGAP were also observed in the hippocampus of WT and $\alpha$-Syn KO mice, compared with their control counterpart mice. Remarkably, we also found that $\alpha$-Syn KO mice relieved the Mn-induced degradation of PSD95, phosphorylated CaMKII $\alpha$, and reduced the level of SynGAP expression (PSD95 protein, $F \quad(\mathrm{Mn} \quad$ factor $)=151.670, \quad P=0.00$, $F$ (genotype) $=0.032, P>0.05, F$ (genotype vs. Mn interaction) $=$ 15.335, $P=0.004 ; \mathrm{p}$-CaMKIIa protein, $F$ (Mn factor $)=$ $364.557, P=0.00, F$ (genotype) $=1.155, P>0.05, F$ (genotype vs. Mn interaction) $=9.767, P=0.014$; SynGAP protein, $F_{(\mathrm{Mn}}$ factor $)=84.375, P=0.00, \quad F \quad$ (genotype) $=1.137, P>0.05$, $F$ (genotype vs. Mn interaction) $=43.539, P=0.002$, Fig. 2 f-i). These results showed that $\alpha$-Syn KO partly relieved Mninduced synaptic degeneration.

\section{Mn-dependent enhanced a-Syn expression disturbs the phosphorylation of NMDA receptors}

To further confirm that $\alpha$-Syn overexpression disturbs the phosphorylation of NMDA receptors, $\alpha$-Syn overexpression and the phosphorylated and total protein levels of GluN2A and GluN2B (the subunits of NMDA receptors) were analyzed in vivo and in vitro. Western blot analysis revealed that the level of $\alpha$-Syn protein was increased in the hippocampus of Mn-treated WT mice (3.25-fold, $P<0.01$, Fig. 3a, b). Between the WT and $\alpha$-Syn KO control groups, statistical analysis showed no differences in the phosphorylated and total protein levels of GluN2A and GluN2B. Following Mn treatment in WT and $\alpha$-Syn KO mice, we found that the phosphorylation levels of GluN2B were strongly increased $(P<0.01$, Fig. 3c, d), and that GluN2A and GluN2B total protein levels were strongly decreased compared with their control counterparts (GluN2B protein, $F$ (Mn factor) $=70.040, P=0.00$, $F_{\text {(genotype) }}=0.117, P>0.05, F$ (genotype vs. Mn interaction) $=$ $0.355, P>0.05$; GluN2A protein, $F$ (Mn factor) $=18.771$, $P=0.003, F$ (genotype) $=0.012, p>0.05, \mathrm{~F}$ (genotype vs. Mn interaction) $=0.014, p>0.05$, Fig. 3e, f). In addition, Western blot analysis showed that the phosphorylation levels of GluN2B were significantly reduced in $\mathrm{Mn}$-treated WT mice compared with Mn-treated $\alpha$-Syn $\mathrm{KO}$ mice (p-GluN2B protein, $F$ (Mn factor) $=487.826, P=0.00, F$ 


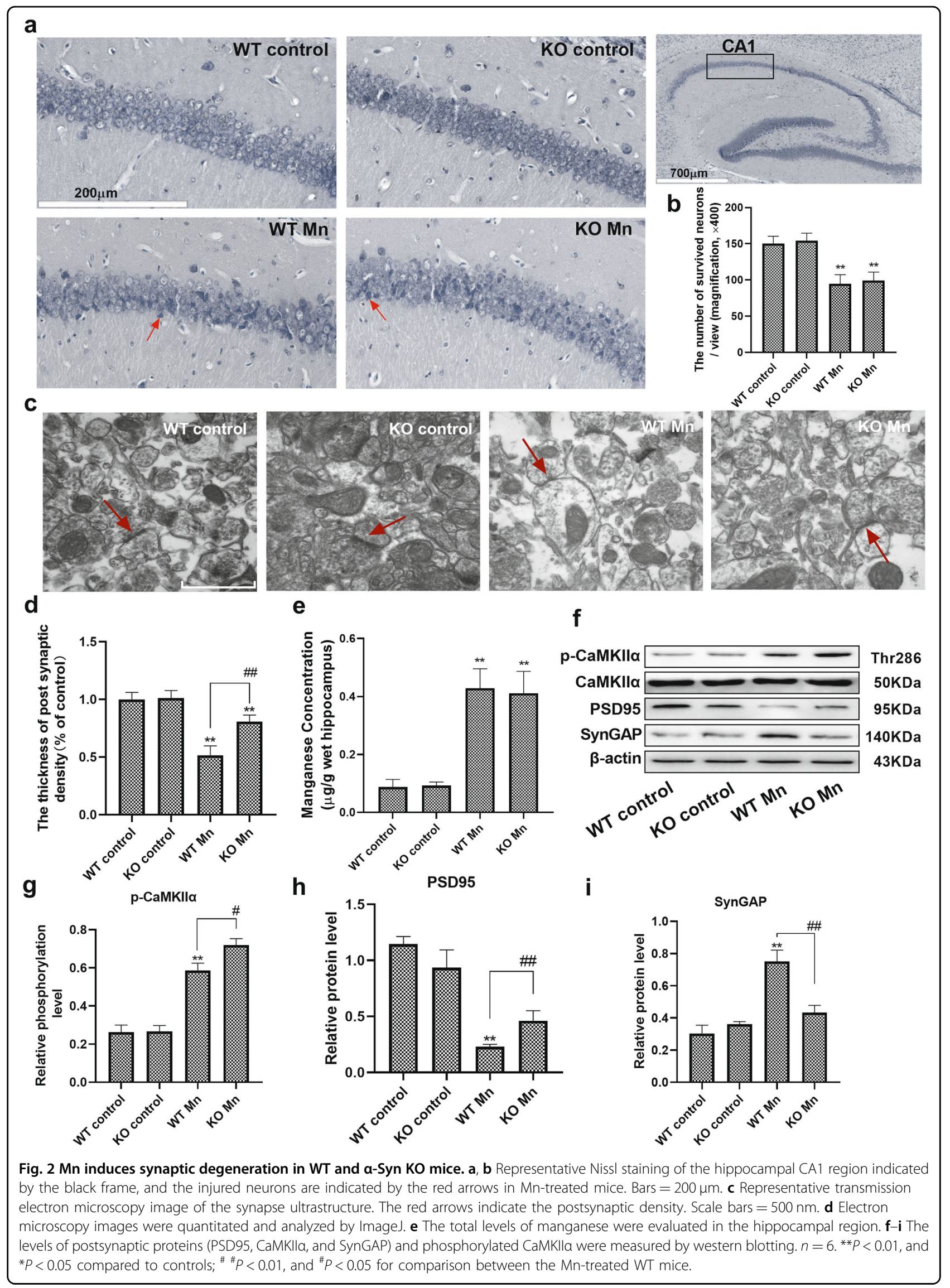




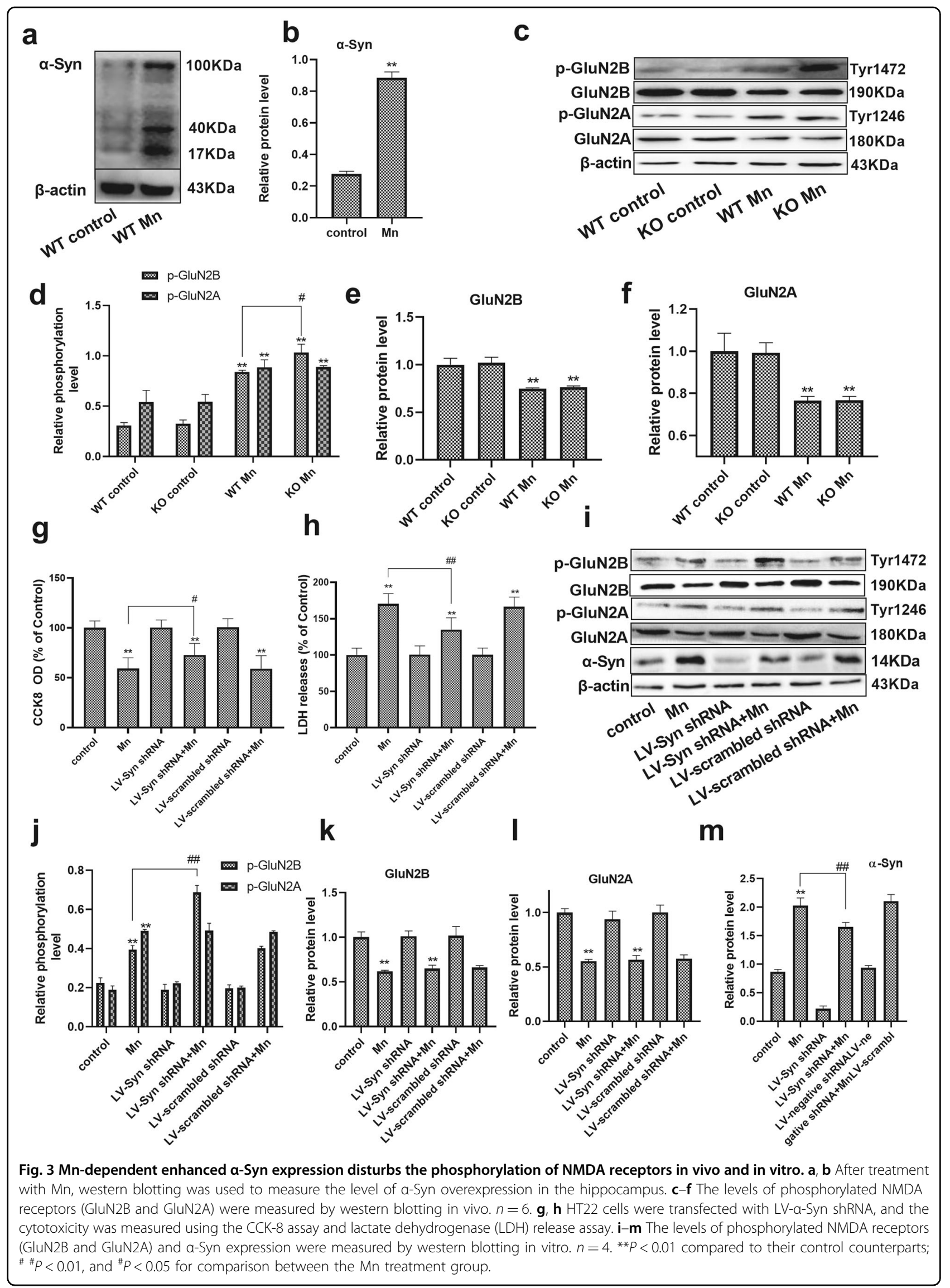




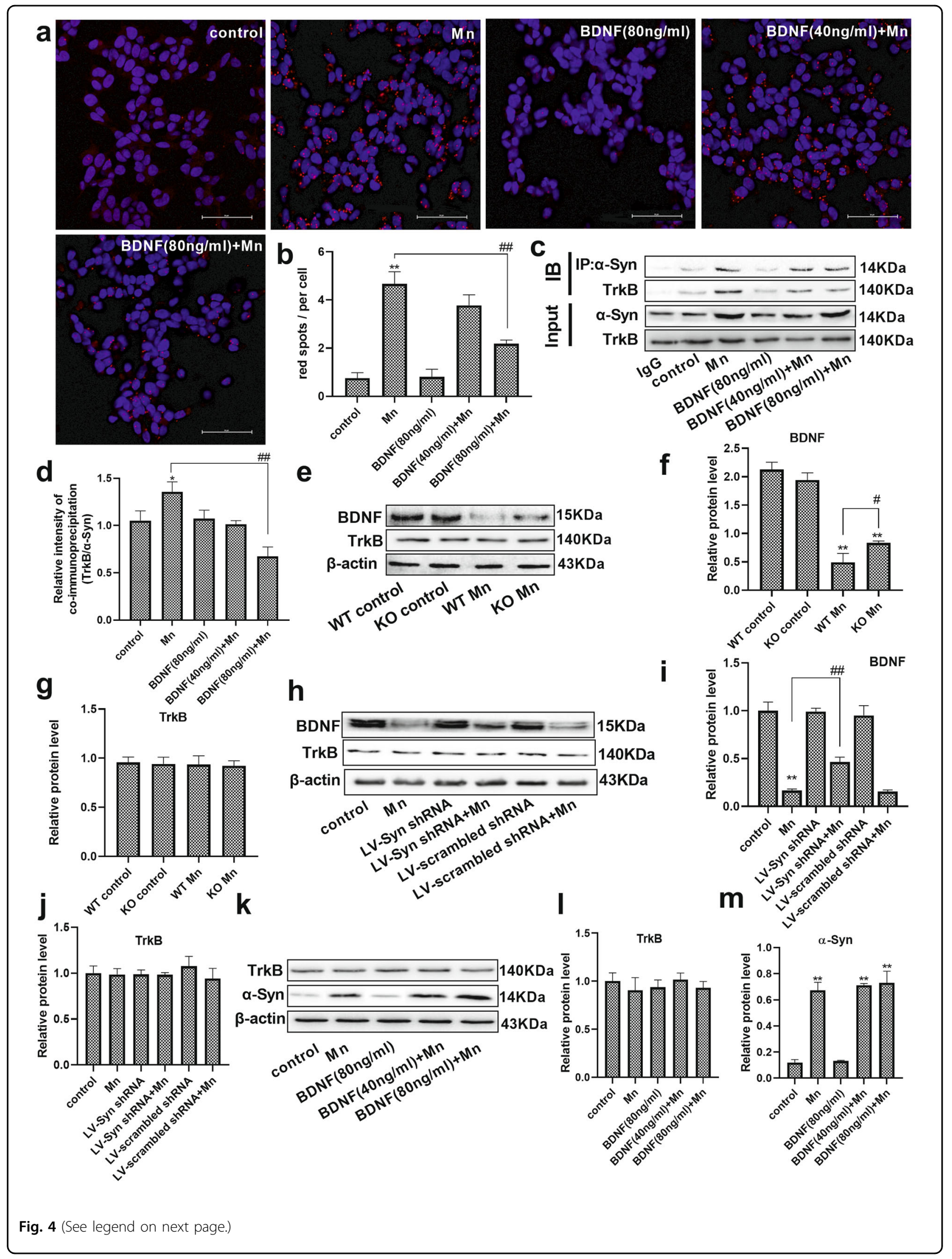


(see figure on previous page)

Fig. 4 Brain-derived neurotrophic factor (BDNF) interfered with the interaction of $\mathbf{a}$-Syn and TrkB. $\mathbf{a}$, b After pretreatment with BDNF and Mn in HT22 cells, representative images of PLA show the interaction between a-Syn and TrkB (red spots), and the nucleus was stained in blue (DAPI). Scale bars $=50 \mu \mathrm{m}$. $\mathbf{c}$, $\mathbf{d}$ The immunoprecipitation analysis of a-Syn and TrkB is shown. $\mathbf{e}-\mathbf{g}$ After treating the mice with Mn, the levels of BDNF and TrkB were evaluated by western blotting. $n=6$. $\mathbf{h}$ - $\mathbf{j}$ For HT22 cells transfected with the LV-a-Syn shRNA, the levels of BDNF and TrkB were evaluated by western blotting after Mn treatment. $\mathbf{k}-\mathbf{m}$ After pretreatment with BDNF and Mn in HT22 cells, the levels of TrkB and a-Syn expression were evaluated by western blotting. $n=4$. ${ }^{*} P<0.01$, and ${ }^{*} P<0.05$ compared to their control counterparts; ${ }^{\# \#} P<0.01$, and ${ }^{\#} P<0.05$ for comparison between the $\mathrm{Mn}$ treatment group.

$($ genotype $)=1.299, P>0.05, F($ genotype vs. $M n$ interaction $)=$ 9.962, $P=0.013)$.

To further verify that the downregulation of phosphorylated GluN2B is associated with Mn-dependent $\alpha$-Syn overexpression, differentiated HT22 (mouse hippocampal neuron precursor) cells were transfected with LV- $\alpha$-Syn shRNA. The cytotoxicity was measured using the CCK- 8 assay, and it was found that there was a significant decrease in Mn-treated cells compared with control cells $(P<0.01$, Fig. $3 g)$. However, pretreatment with LV- $\alpha$-Syn shRNA increased cell viability (1.23-fold, $P<0.05)$ and decreased LDH release compared with cells treated with $\mathrm{Mn}$ alone $(20.78 \%, P<0.01$, Fig. 3h). Similarly, western blot analysis results showed that $\mathrm{Mn}$ treatment increased the phosphorylation levels $(P<0.01$, Fig. 3i, j) and decreased total protein levels of GluN2A and GluN2B compared with control cells $(P<0.01$, Fig. $3 k, 1)$. The phosphorylation levels of GluN2B in cells pretreated with LV- $\alpha$-Syn shRNA were significantly higher than those in the cells treated with Mn alone (1.74fold, $P<0.01)$. In addition, the level of $\alpha$-Syn expression was increased in Mn-treated cells compared with control cells $(P<0.01)$. Pretreatment with LV- $\alpha$-Syn shRNA decreased the level of $\alpha$-Syn expression compared with cells treated with $\mathrm{Mn}$ alone $(P<0.01$, Fig. $3 \mathrm{~m})$. These results suggested that $\mathrm{Mn}$-dependent enhanced $\alpha$-Syn expression was responsible for the downregulation of phosphorylated GluN2B.

\section{BDNF interferes with the interaction of a-Syn and TrkB}

We hypothesized that Mn-dependent enhanced $\alpha$-Syn expression might directly impinge on the BDNF/TrkB pathway. Therefore, we conducted a proximity ligation assay (PLA) using differentiated HT22 cells and found that there was an increasing proximity ligation (PL) signal between $\alpha$-Syn and TrkB in Mn-treated cells compared with the control cells (6.21-fold, $P<0.01$, Fig. 4a, b). As expected, we also found that the same tendency was observed in the co-immunoprecipitation (Co-IP) assay (Fig. 4c). The semiquantitative analysis showed that $\mathrm{Mn}$ exposure significantly increased the interaction of $\alpha$-Syn and TrkB compared with the control group $(P<0.05$, Fig. 4d).
Next, we investigated whether exogenous BDNF could disrupt the interaction of $\alpha$-Syn and TrkB and protect HT22 cells from damage. The cytotoxicity was measured using the CCK- 8 assay and LDH release assay, and it was found that pretreatment with $80 \mathrm{ng} \mathrm{ml}^{-1}$ BDNF increased neuronal cell viability (1.37-fold, $P<0.01$, Supplementary Fig. s1a) and decreased LDH release compared with cells treated with $\mathrm{Mn}$ alone $(20.05 \%, P<0.01$, Supplementary Fig. s1b). In addition, we found that $80 \mathrm{ng} \mathrm{ml}^{-1}$ BDNF pretreatment significantly decreased the interaction of $\alpha$ Syn and TrkB compared with cells treated with Mn alone (50.55\%, $P<0.01)$. These results suggested that $\alpha$-Syn interacted with TrkB, which could be inhibited by BDNF treatment.

To investigate the effect of $\alpha$-Syn on BDNF and the TrkB receptor in response to $\mathrm{Mn}$-associated pathology, we next treated mice and differentiated HT22 cells with Mn, LV- $\alpha$-Syn shRNA, and BDNF, and analyzed the levels of $\alpha$-Syn, BDNF, and TrkB expression. After treatment with $\mathrm{Mn}$, a significant decrease in the level of BDNF expression was observed in the hippocampus of WT and $\alpha$-Syn KO mice, compared with their control counterpart mice $\quad(B D N F, \quad F \quad(\mathrm{Mn} \quad$ factor $)=380.508, \quad P=0.00$, $F_{\text {(genotype) }}=1.277, P>0.05, F$ (genotype vs. Mn interaction) $=$ 3.993, $P=0.016$ ). Remarkably, we also found that the effect of Mn on BDNF expression was weaker in $\alpha$-Syn KO mice compared to WT mice $(P<0.05$, Fig. 4e, f). However, neither $\mathrm{Mn}$ treatment nor $\alpha$-Syn $\mathrm{KO}$ affected the level of TrkB expression (Fig. 4g).

To further verify the effect of Mn-dependent enhanced $\alpha$-Syn expression on BDNF and the TrkB receptor, cells were transfected with LV- $\alpha$-Syn shRNA. Similarly, western blot analysis indicated that Mn treatment decreased the level of BDNF expression compared with the control cells $(P<0.01$, Fig. $4 \mathrm{~h}, \mathrm{i})$. In addition, after cells were treated with $\mathrm{Mn}$, the level of BDNF in cells pretreated with LV- $\alpha$-Syn shRNA was significantly higher than that in the normal cells $(2.79$-fold, $P<0.01)$. Western blot results showed that there was no significant difference in the level of TrkB expression among the experimental groups (Fig. 4j). Furthermore, BDNF pretreatment did not affect the levels of TrkB and $\alpha$-Syn expression compared with Mn-treated cells (Fig. $4 \mathrm{k}-\mathrm{m}$ ). 


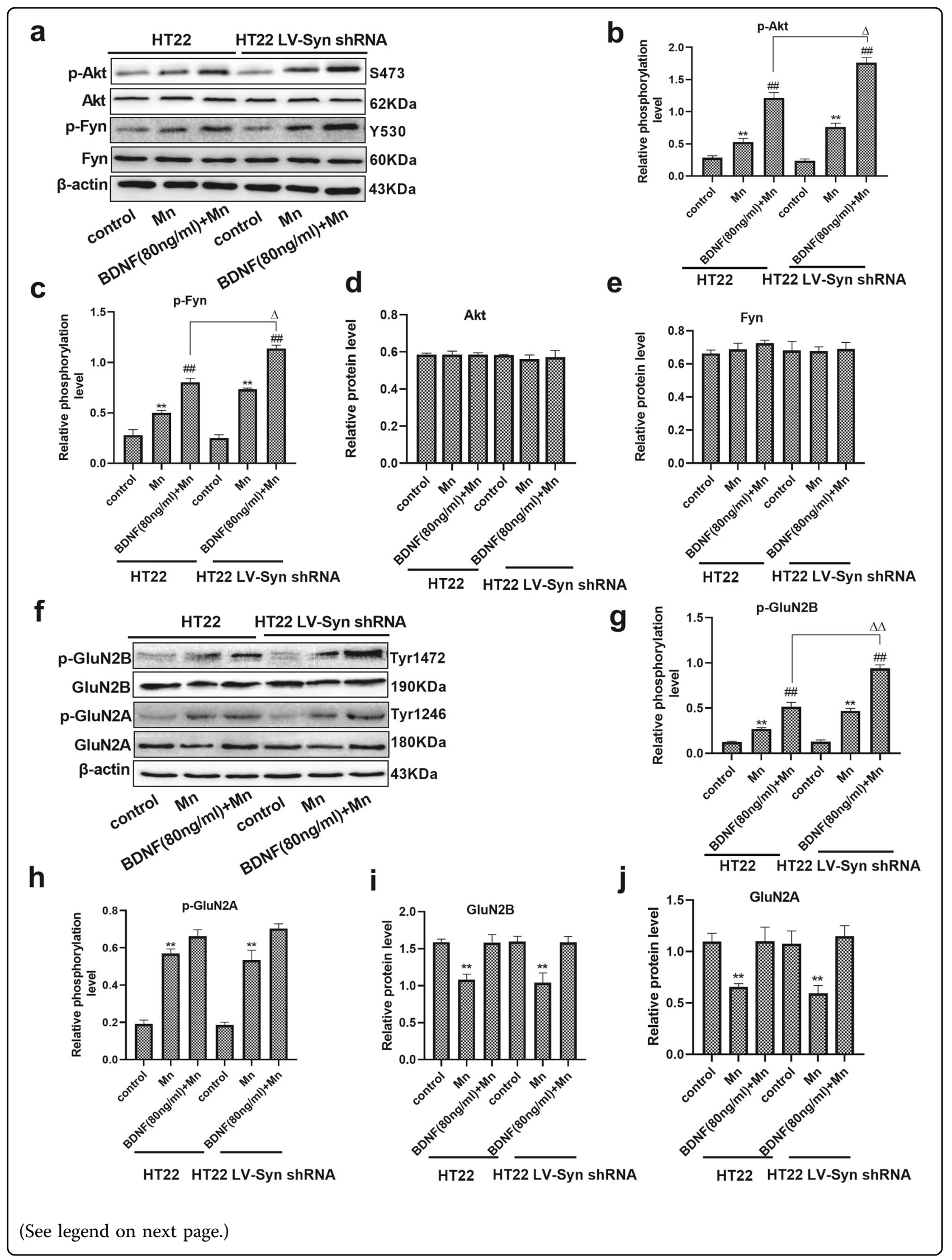


(see figure on previous page)

Fig. 5 TrkB/Akt/Fyn signaling is required for the phosphorylation of NMDA receptors in vitro. a-e After normal or transfected HT22 cells were pretreated with brain-derived neurotrophic factor (BDNF) and Mn, the levels of phospho-Akt and phospho-Fyn, and Akt and Fyn expression were evaluated by western blotting. $\mathbf{f}-\mathbf{j}$ After normal or transfected HT22 cells were pretreated with BDNF and Mn, the levels of phospho-GluN2B and phospho-GluN2A, and the expression of GluN2B and GluN2A were evaluated by western blotting. $n=4$. $\beta$-actin was used as a loading control. ** $P<$ 0.01 , and ${ }^{*} P<0.05$ compared to their control counterparts; ${ }^{\# \#} P<0.01$ compared to their Mn-treated cells; ${ }^{\Delta} P<0.01$, and ${ }^{\Delta} P<0.05$ for comparison between their BDNF-pretreated and Mn-treated cells.

\section{TrkB/Akt/Fyn signaling is required for the phosphorylation} of NMDA receptors

To examine the biological effect of $\alpha$-Syn on BDNF/ TrkB signaling, we transfected differentiated HT22 cells with LV- $\alpha$-Syn shRNA, followed by BDNF and Mn stimulation. In differentiated HT22 cells, exposure to Mn resulted in an increase in the phosphorylation levels of Akt and Fyn compared with their controls (1.87- and 1.79 -fold, respectively, $P<0.01$, Fig. 5a-e). After pretreatment with BDNF, the phosphorylation levels of Akt and Fyn were evaluated. The results clearly showed that phosphorylation levels of these proteins were upregulated compared with those in cells treated with Mn (2.29- and 1.61 -fold, respectively, $P<0.01)$. Remarkably, we also found that BDNF-pretreated and Mn-treated cells with LV- $\alpha$-Syn shRNA showed an increase in the phosphorylation levels of Akt and Fyn compared with BDNFpretreated and Mn-treated HT22 cells (1.45- and 1.41fold, respectively, $P<0.05)$, and also a decrease compared with alone BDNF-treated cells with LV- $\alpha$-Syn shRNA ( $P$ $<0.01$, Supplementary Fig. s2a-e).

To further verify that BDNF/TrkB signaling contributed to the phosphorylation of GluN2A and GluN2B, BDNF was added to Mn-treated HT22 cells. Western blot analysis results showed that in BDNF-pretreated and Mntreated cells with LV- $\alpha$-Syn shRNA group, the phosphorylated protein levels of GluN2B were upregulated (1.82-fold, $P<0.01$, Fig. 5f-h) compared with BDNFpretreated and Mn-treated HT22 cells, and the total protein levels of GluN2A and GluN2B were significantly decreased $(P<0.01$, Fig. 5i, j) compared with their control counterparts. However, statistical analysis showed no difference in the phosphorylation level of GluN2A in BDNF-pretreated normal or transfected HT22 cells. Taken together, these results indicate that Mn-dependent enhanced $\alpha$-Syn expression was responsible for the downregulation of phosphorylated GluN2B through the inhibition of BDNF-mediated TrkB signaling.

\section{Discussion}

The hippocampus, as a long-term memory and learning region, is one of the most sensitive areas to Mn overexposure in the brain ${ }^{20}$. In this regard, this study investigated the mechanisms of learning and memory impairments via modulating the signaling pathway factors in the hippocampus after exposure to Mn. In this study, we highlight two significant observations. One is that Mn causes impairments in learning and memory and the inhibition of LTP, which are related to the phosphorylation levels of NMDA receptors. The other is that Mndependent enhanced $\alpha$-Syn expression and BDNF reduction disturbed $\operatorname{TrkB}$ signaling, resulting in the downregulation of phosphorylated GluN2B.

In this study, we found that no difference existed between genotypes in spatial memory function in the control groups. However, when treated by Mn, both WT and $\alpha$-Syn $\mathrm{KO}$ mice showed worse performance in both the acquisition and removal phase compared with the control groups. These results may represent a manifestation of learning and memory impairments in $\mathrm{Mn}$ treated mice. In addition, our results indicated that $\alpha$-Syn KO might ameliorate spatial memory impairment caused by $\mathrm{Mn}$. However, there was no difference in speed between genotypes in the training or testing phase, which partially indicated the emotional influences on the outcomes of the water maze test. The formation of LTP is considered as a potential cellular mechanism in learning and memory processes. According to the findings of previous studies ${ }^{21}$, our study revealed that $\alpha$-Syn KO did not influence the LTP under normal conditions. This might be because LTP is mainly caused by postsynaptic mechanisms in the CA3-CA1 pathway, but the $\alpha$-Syn effects on synaptic transmission are predominantly presynaptic $^{22}$. However, Mn exposure promotes $\alpha$-Syn secretion in exosomal vesicles, which subsequently evokes proinflammatory and neurodegenerative responses in both cell culture and animal models ${ }^{23}$, and thus, it can be supposed that the exosome-mediated, cell-to-cell transmission of $\alpha$-Syn during exposure to Mn may inhibit the formation of LTP leading to the regression of spatial memory in Mn-treated mice.

The greater selective neuron loss and synaptic degeneration in the hippocampus after Mn treatment may account for the spatial memory and LTP impairments. As shown in this study, surviving neurons were stained by Nissl in the CA1 region, which was important for LTP induction, and these were significantly decreased in Mntreated WT and $\alpha$-Syn KO mice. In addition, the synaptic ultrastructural alterations produced by the neurotoxic effect of Mn in the hippocampus were the decrease in the 


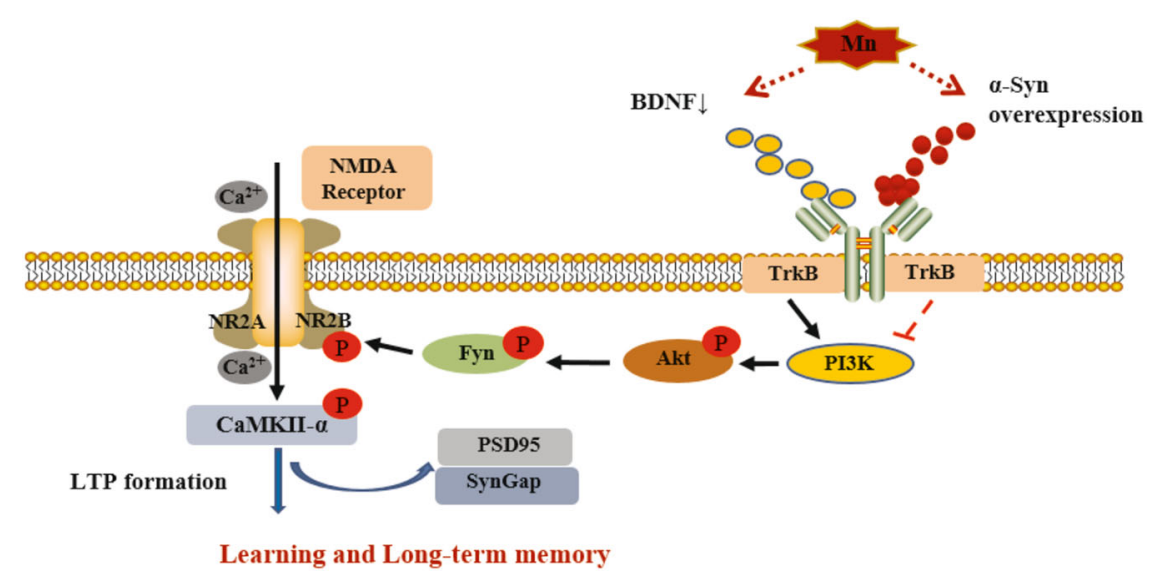

Fig. 6 The proposed mechanism of Mn-induced spatial memory and long-term potentiation (LTP) impairments. Mn-induced a-Syn protein overexpression contributes to further exacerbate brain-derived neurotrophic factor (BDNF) protein-level reduction and to inhibit TrkB/Akt/Fyn signaling, thereby disturbing Fyn-mediated phosphorylation of the NMDA receptor GluN2B subunit at tyrosine.

thickness of PSD, while $\alpha$-Syn knockout weakened the effect of Mn. An elevation of Mn concentration in the hippocampus was detected in Mn-treated mice. Consistent with this notion, the elevation of Mn concentration in the hippocampus resulted in deficits in spatial memory, loss of neurons, and impairment in hippocampal $\mathrm{LTP}^{21,24}$. However, between Mn-treated WT and $\alpha$-Syn KO mice, no significant difference was shown in neuronal injury or Mn concentration in the hippocampus. The major reason for this is that multiple factors are involved in $\mathrm{Mn}$ induced neurotoxicity, including oxidative injury, mitochondrial dysfunction, and neuroinflammation ${ }^{1}$.

A few studies have proved that postsynaptic signaling proteins, such as PSD95 $\mathrm{Ca}^{2+} /$ calmodulin-dependent protein kinase II with alpha chain (CaMKII $)$, and synaptic Ras GTPase-activating protein (SynGAP) associated with NMDA receptors in the hippocampus, play a vital role in $\mathrm{LTP}^{11}$. Downregulation of the levels of PSD95 and phosphorylated CaMKII $\alpha$ related to enhancing the expression of SynGAP upon exposure to Mn as shown in this study could be associated with an alteration in LTP. Consistent with the findings in LTP impairments, $\alpha$-Syn $\mathrm{KO}$ relieved Mn-induced the degradation of PSD95, phosphorylated CaMKII $\alpha$, and reduced the level of SynGAP expression.

The N-methyl-D-aspartate (NMDA) receptor is a heteromeric complex of three subunits differentially distributed in the brain. Some studies showed that phosphorylation of the NMDA receptor 2 subunit (NR2), especially GluN2A and GluN2B, regulates the activity of NMDA receptors and induces LTP formation, memory formation, and synaptic plasticity ${ }^{25}$. Our previous study also showed that calpain was activated by Mn-mediated $\mathrm{Ca}^{2+}$ influx and exhibited selectivity in the degradation of GluN2A and GluN2B $B^{17,26}$. In this study, a decrease in the total protein levels of both GluN2A and GluN2B was observed on $\mathrm{Mn}$ exposure both in vivo and in vitro, and these results were consistent with our previous findings ${ }^{27}$. In particular, phosphorylated GluN2B has been shown to play a vital role in NMDA receptor activation. In this study, Mn-treated mice exhibited elevation of phosphorylated NMDA receptor, while the phosphorylation level of GluN2B was significantly lower in Mn-treated WT mice compared with Mn-treated $\alpha$-Syn KO mice; this downregulation caused the decline of LTP in Mn-treated WT mice. Additionally, HT22 cells transfected with LV$\alpha$-Syn shRNA exhibited an elevation of phosphorylated GluN2B induced by Mn treatment. The phosphorylation level of GluN2A did not differ between normal and LV- $\alpha$ Syn shRNA-transfected cells. These results suggested that $M n$-dependent enhanced $\alpha$-Syn expression participated in NMDA receptor activation, especially through the phosphorylation of GluN2B.

BDNF is a potent dopaminergic neurotrophin involved in regulating NMDA receptor-dependent synaptic plasticity $^{28}$. In this study, we found that $\mathrm{Mn}$ treatment decreased the level of BDNF expression in both WT and $\alpha$-Syn $\mathrm{KO}$ mice. In addition, there was a more significant decline in BDNF of Mn-treated WT mice, while Mn treatment resulted in $\alpha$-Syn protein overexpression. These results are consistent with previous findings ${ }^{29}$ and suggest that $\alpha$-Syn overexpression might account for the downregulation of BDNF. Therefore, it is highly possible that BDNF downregulation might have a profound effect on the inhibition of BDNF/TrkB signaling. Studies have indicated that many important cellular processes, such as synaptic vesicle recycling, intracellular trafficking, mitochondrial energetics, lysosomal activity, and autophagy, are all susceptible to $\alpha$-Syn toxicity ${ }^{30,31}$, suggesting a multifaceted mode of action of neuronal toxicities by the 
accumulation of $\alpha$-Syn. Due to the well-documented correlation between aberrant $\alpha$-Syn accumulation and neuronal degeneration, we hypothesized that pathological $\alpha$-Syn might directly impinge on the BDNF/TrkB pathway. To test this hypothesis, we conducted PLA and CoIP assay and found that $\alpha$-Syn selectively interacted with TrkB, which was relieved by exogenous BDNF pretreatment. Hence, this innovative finding provides insight into a pathological role(s) of $\alpha$-Syn in mediating BDNF/TrkB signal transduction and may represent an obscure mechanism by which a-Syn contributes to spatial memory and LTP impairments.

BDNF and the activation of its high-affinity receptor TrkB play a vital role in the neuroprotection mediated by the PI3K/Akt signaling pathway. The PI3K/Akt signaling pathway is commonly activated by oxidative stress against nerve cell death ${ }^{32}$. In our study, overexposure to $\mathrm{Mn}$ triggered the PI3K/Akt pathway through upregulating the phosphorylation level of Akt, which then inhibited the transcription function of apoptosis genes and negatively regulated apoptosis, leading to cell survival ${ }^{33}$. Interestingly, a significant increase in the level of p-Akt expression was observed in cells transfected with LV- $\alpha$-Syn shRNA, followed by BDNF stimulation. These findings indicated that $\alpha$-Syn disturbed BDNF/TrkB signal transduction. Fyn, a member of the Src family, has been proved to mediate phosphorylation and upregulate GluN2Bcontaining NMDA receptors, leading to enhanced receptor channel activity ${ }^{34}$. As a downstream signaling molecule of Akt, Fyn kinase is essential for synaptic plasticity ${ }^{35,36}$. In our study, a significant increase in the level of p-Fyn had been observed in cells transfected with LV- $\alpha$-Syn shRNA, followed by BDNF stimulation compared with Mn-treated HT22 cells, which was consistent with the phosphorylation of GluN2B. These results are identical to previous findings ${ }^{37}$, and suggest that Fynmediated phosphorylation of GluN2B acts on the tyrosine 1472 (Tyr1472) site and plays an important role in hippocampal LTP and hippocampus-dependent memory formation. However, the deletion of $\alpha$-Syn does not entirely annul the effects of Mn on Fyn-mediated phosphorylation of GluN2B. This may be explained by other mechanisms of $\mathrm{Mn}$ including accelerating BDNF protein degradation and reducing binding to $\operatorname{TrkB}^{3,38}$, which contribute to $\mathrm{Mn}$-induced neurotoxicity.

\section{Conclusion}

This study provided evidence that Mn-induced $\alpha$-Syn protein overexpression contributes to further exacerbate BDNF protein-level reduction and to inhibit TrkB/Akt/ Fyn signaling, thereby disturbing Fyn-mediated phosphorylation of the NMDA receptor GluN2B subunit at tyrosine (Fig. 6). In KO $\alpha$-Syn mice treated with $\mathrm{Mn}$, spatial memory and LTP impairments were less pronounced than in WT mice. However, the same robust neuronal death was observed in the face of Mn-induced neurotoxicity.

\section{Acknowledgements}

This study was funded by National Natural Science Foundation of China (81372942).

\section{Data availability}

All data generated or analyzed during this study are included in this published article.

\section{Conflict of interest}

The authors declare that they have no conflict of interest.

\section{Ethics approval and consent to participate}

All experiments were conducted in consistent with the experimental animal administration committee of China Medical University (approval no.

CMU2017145) for the care and use of laboratory animals and efforts were made to minimize their suffering.

\section{Publisher's note}

Springer Nature remains neutral with regard to jurisdictional claims in published maps and institutional affiliations.

Supplementary Information accompanies this paper at (https://doi.org/ 10.1038/s41419-020-03051-2).

Received: 12 January 2020 Revised: 24 September 2020 Accepted: 28 September 2020

Published online: 08 October 2020

\section{References}

1. Harischandra, D. S. et al. Manganese-induced neurotoxicity: new insights into the triad of protein misfolding, mitochondrial impairment, and neuroinflammation. Front. Neurosci. 13, 654 (2019).

2. Kullar, S. S. et al. A benchmark concentration analysis for manganese in drinking water and IQ deficits in children. Environ. Int. 130, 104889 (2019).

3. Bailey, R. A. et al. Effects of preweaning manganese in combination with adult striatal dopamine lesions on monoamines, BDNF, TrkB, and cognitive function in Sprague-Dawley rats. Neurotox. Res 35, 606-620 (2019).

4. Erikson, K. M. \& Aschner, M. Manganese: its role in disease and health. Met. Ions Life Sci 19, 253-266 (2019).

5. Xu, Y. et al. BDNF activates TrkB/PLCgamma1 signaling pathway to promote proliferation and invasion of ovarian cancer cells through inhibition of apoptosis. Eur. Rev. Med. Pharm. Sci. 23, 5093-5100 (2019).

6. Numakawa, T. et al. BDNF function and intracellular signaling in neurons. Histol. Histopathol. 25, 237-258 (2010).

7. Kang, S. S. et al. TrkB neurotrophic activities are blocked by alpha-synuclein, triggering dopaminergic cell death in Parkinson's disease. Proc. Natl Acad. Sci. USA 114, 10773-10778 (2017).

8. Yan, D. Y. et al. Mn-induced neurocytes injury and autophagy dysfunction in alpha-synuclein wild-type and knock-out mice: highlighting the role of alphasynuclein. Neurotox. Res. 36, 66-80 (2019).

9. Teravskis, P. J. et al. A53T mutant alpha-synuclein induces tau-dependent postsynaptic impairment independently of neurodegenerative changes. J. Neurosci. 38, 9754-9767 (2018).

10. $\mathrm{Xu}, \mathrm{B}$. et al. alpha-Synuclein is involved in manganese-induced ER stress via PERK signal pathway in organotypic brain slice cultures. Mol. Neurobiol. 49, 399-412 (2014).

11. Shentu, Y. P. et al. CIP2A-promoted astrogliosis induces AD-like synaptic degeneration and cognitive deficits. Neurobiol. Aging 75, 198-208 (2019).

12. Liu, M. G. et al. Acid-sensing ion channel 1a contributes to hippocampal LTP inducibility through multiple mechanisms. Sci. Rep. 6, 23350 (2016). 
13. Zhou, Q. et al. Adenosine A1 receptors play an important protective role against cognitive impairment and long-term potentiation inhibition in a pentylenetetrazol mouse model of epilepsy. Mol. Neurobiol. 55, 3316-3327 (2018).

14. Wang, C. et al. Inhibition of calpains protects Mn-induced neurotransmitter release disorders in synaptosomes from mice: involvement of SNARE complex and synaptic vesicle fusion. Sci. Rep. 7, 3701 (2017).

15. Bonaterra, G. A. et al. Neurotrophic, cytoprotective, and anti-inflammatory effects of St. John's wort extract on differentiated mouse hippocampal HT-22 neurons. Front. Pharm. 8, 955 (2017).

16. Liu, D. M. et al. RNA interference mediated silencing of alpha-synuclein in MN9D cells and its effects on cell viability. Neurosci. Bull. 24, 96-104 (2008).

17. Wang, C. et al. Alpha-synuclein and calpains disrupt SNARE-mediated synaptic vesicle fusion during manganese exposure in SH-SY5Y cells. Cells 7, 258 (2018).

18. Ma, Z. et al. The role S-nitrosylation in manganese-induced autophagy dysregulation in SH-SY5Y cells. Environ. Toxicol. 32, 2428-2439 (2017).

19. Sandison, H. E. et al. PLK1 and $Y Y 1$ interaction in follicular lymphoma is associated with unfavourable outcome. J. Clin. Pathol. 66, 764-767 (2013).

20. Pereda, D., Al-Osta, I., Okorocha, A. E., Easton, A. \& Hartell, N. A. Changes in presynaptic calcium signalling accompany age-related deficits in hippocampal LTP and cognitive impairment. Aging Cell 18, e13008 (2019).

21. Amos-Kroohs, R. M. et al. Developmental manganese neurotoxicity in rats: cognitive deficits in allocentric and egocentric learning and memory. Neurotoxicol Teratol. 59, 16-26 (2017).

22. Atias, M. et al. Synapsins regulate alpha-synuclein functions. Proc. Natl Acad. Sci. USA 116, 11116-11118 (2019).

23. Harischandra, D. S. et al. Manganese promotes the aggregation and prion-like cell-to-cell exosomal transmission of alpha-synuclein. Sci. Signal 12, eaau4543 (2019).

24. Carvalho, C. F. et al. Environmental manganese exposure and associations with memory, executive functions, and hyperactivity in Brazilian children. Neurotoxicology 69, 253-259 (2018).

25. Nakai, T. et al. Girdin phosphorylation is crucial for synaptic plasticity and memory: a potential role in the interaction of BDNF/TrkB/Akt signaling with NMDA receptor. J. Neurosci. 34, 14995-15008 (2014).
26. Zhao, J., Baudry, M. \& Jones, S. Calpain inhibition reduces NMDA receptor rundown in rat substantia nigra dopamine neurons. Neuropharmacology 137, 221-229 (2018)

27. $\mathrm{Xu}, \mathrm{B} . \mathrm{Xu}, \mathrm{Z}$. F. \& Deng, Y. Effect of manganese exposure on intracellular $\mathrm{Ca}^{2+}$ homeostasis and expression of NMDA receptor subunits in primary cultured neurons. Neurotoxicology 30, 941-949 (2009).

28. Marcos, J. L. et al. Role of the spinal TrkB-NMDA receptor link in the BDNFinduced long-lasting mechanical hyperalgesia in the rat: a behavioural study. Eur. J. Pain. 21, 1688-1696 (2017)

29. Yuan, Y. et al. Overexpression of alpha-synuclein down-regulates BDNF expression. Cell Mol. Neurobiol. 30, 939-946 (2010).

30. Zhang, G. et al. New perspectives on roles of alpha-synuclein in Parkinson's disease. Front. Aging Neurosci. 10, 370 (2018).

31. Ottolini, D., Cali, T., Szabo, I. \& Brini, M. Alpha-synuclein at the intracellular and the extracellular side: functional and dysfunctional implications. Biol. Chem. 398, 77-100 (2017)

32. Linton, M. F., Moslehi, J. J. \& Babaev, V. R. Akt signaling in macrophage polarization, survival, and atherosclerosis. Int. J. Mol. Sci. 20, 2703 (2019).

33. Cheng, $\mathrm{H}$. et al. PI3K/Akt signaling pathway and Hsp70 activate in hippocampus of rats with chronic manganese sulfate exposure. J. Trace Elem. Med. Biol. 50, 332-338 (2018).

34. Trepanier, C. H., Jackson, M. F. \& MacDonald, J. F. Regulation of NMDA receptors by the tyrosine kinase Fyn. FEBS J. 279, 12-19 (2012).

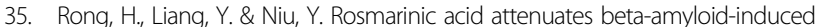
oxidative stress via Akt/GSK-3beta/Fyn-mediated Nrf2 activation in PC12 cells. Free Radic. Biol. Med. 120, 114-123 (2018).

36. Mathur, A., Rizvi, F. \& Kakkar, P. PHLPP2 down regulation influences nuclear Nrf2 stability via Akt-1/Gsk3beta/Fyn kinase axis in acetaminophen induced oxidative renal toxicity: protection accorded by morin. Food Chem. Toxicol. 89, 19-31 (2016).

37. Li, S. et al. BDNF contributes to spinal long-term potentiation and mechanical hypersensitivity via Fyn-mediated phosphorylation of NMDA receptor GluN2B subunit at tyrosine 1472 in rats following spinal nerve ligation. Neurochem Res. 42, 2712-2729 (2017)

38. Zhu, G. et al. PKA- and Ca(2+)-dependent p38 MAPK/CREB activation protects against manganese-mediated neuronal apoptosis. Toxicol. Lett. 309, 10-19 (2019). 